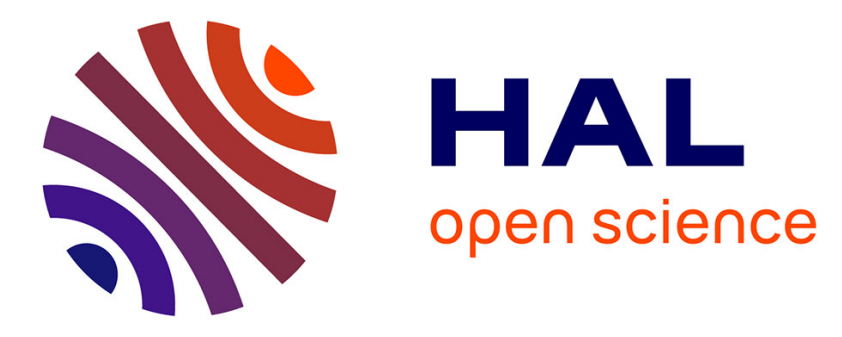

\title{
System-Level Optimization in Poisson Cellular Networks: An Approach Based on the Generalized Benders Decomposition
}

Jian Song, Marco Di Renzo, Alessio Zappone, Vincenzo Sciancalepore, Xavier Costa Perez

\section{To cite this version:}

Jian Song, Marco Di Renzo, Alessio Zappone, Vincenzo Sciancalepore, Xavier Costa Perez. SystemLevel Optimization in Poisson Cellular Networks: An Approach Based on the Generalized Benders Decomposition. IEEE Wireless Communications Letters, 2020, 9 (10), pp.1773 - 1777. 10.1109/LWC.2020.3004193 . hal-03020437

\section{HAL Id: hal-03020437 https://hal.science/hal-03020437}

Submitted on 24 Nov 2020

HAL is a multi-disciplinary open access archive for the deposit and dissemination of scientific research documents, whether they are published or not. The documents may come from teaching and research institutions in France or abroad, or from public or private research centers.
L'archive ouverte pluridisciplinaire HAL, est destinée au dépôt et à la diffusion de documents scientifiques de niveau recherche, publiés ou non, émanant des établissements d'enseignement et de recherche français ou étrangers, des laboratoires publics ou privés. 


\title{
System-Level Optimization in Poisson Cellular Networks: An Approach Based on the Generalized Benders Decomposition
}

\author{
Jian Song, Marco Di Renzo, Fellow, IEEE, Alessio Zappone, Senior Member, IEEE, Vincenzo Sciancalepore, \\ Senior Member, IEEE, and Xavier Costa Perez, Senior Member, IEEE
}

\begin{abstract}
During the last decade, stochastic geometry has been widely employed for system-level analysis in cellular networks. The resulting analytical frameworks are, however, not always amenable for system-level optimization. This is due to three main reasons: (i) the performance metric of interest may not be formulated in closed-form; (ii) under some analytically tractable modeling assumptions, important system parameters may not explicitly appear in the analytical frameworks; and (iii) the optimization problem may not possess any structural properties, e.g., convexity, that facilitate the development of numerical algorithms for optimizing multiple (continuous- and discretevalued) parameters at an affordable computational complexity and with performance-guarantee, e.g., the convergence to the global optimum is provable. In this letter, we leverage the new definition of coverage probability in [1], we show that it is suitable to formulate mixed-integer non-linear system-level resource allocation problems in Poisson cellular networks, and we prove that the global optimum can be efficiently calculated by applying the generalized Benders decomposition. Numerical results are illustrated in order to compare the proposed approach against brute-force and greedy-like optimization algorithms.
\end{abstract}

Index Terms-Cellular networks, stochastic geometry, systemlevel analysis, mixed-integer non-linear optimization.

\section{INTRODUCTION}

Stochastic geometry is a widely employed analytical tool for modeling and analyzing the performance of cellular networks [2]. The Poisson Point Process (PPP) is, in particular, the most analytically tractable abstraction for modeling cellular networks. For example, homogeneous and inhomogeneous PPPs have been used for modeling the locations of the Base Stations (BSs) in cellular networks [2], [3], and Boolean schemes of rectangles, whose centers are distributed according to a PPP, have been used for modeling blocking objects [4].

With the aid of stochastic geometry, relevant key performance metrics that quantify the performance of cellular networks, e.g., the spectral efficiency, can be formulated in computable analytical expressions. As recently elaborated in [1], however, the resulting analytical frameworks are not always conveniently formulated for system-level optimization. This originates, in particular, from two main reasons: (i) the performance metric of interest may not be formulated in closedform, which may result in a difficult function to optimize; and (ii) under analytically tractable modeling assumptions, some system parameters may not explicitly appear in the analytical frameworks, which may lead to meaningless optimization problems. Motivated by these considerations, the authors of [1]

Manuscript received XXXXX; revised XXXXXX; accepted XXXXXXXX This work was supported in part by the European Commission through the H2020 5Gaura project under grant number 675806. The associate editor coordinating the review of this article and approving it for publication was H. Dhillon. (Corresponding author: Marco Di Renzo.)

J. Song and M. Di Renzo are with Université Paris-Saclay, CNRS, CentraleSupélec, Laboratoire des Signaux et Systèmes, Gif-sur-Yvette, France.

A. Zappone is with University of Cassino and Southern Lazio, Italy.

V. Sciancalepore and X. Costa-Perez are with NEC Laboratories, Germany.

Digital Object Identifier XXX/LWC.2020.XXX have introduced a new definition of coverage probability that is suitable to formulate meaningful optimization problems. In [1], for example, the optimal transmit power and deployment density of the BSs that maximize the energy efficiency are computed. More recently, the same authors have leveraged the framework in [1] to prove the impact of several design parameters on the coverage [5], and to compute the energyspectral efficiency Pareto front in cellular networks [6].

In spite of these promising results, the authors of [1], [5], [6] have tackled the optimization of a single parameter, e.g., the transmit power or the density of the BSs, and have unveiled the complexity of solving multi-parameter optimization problems (e.g., to optimize the transmit power and the density of the BSs). Also, they have shown that it is often difficult to prove even the existence of an optimizer for every set of parameters. From these research works, we can conclude that it is difficult, in general, to prove structural properties (e.g., the convexity) of optimization problems that originate from stochastic geometry models and analysis. This is, however, needed in order to facilitate the development of numerical algorithms for optimizing multiple (continuous- and discretevalued) parameters at an affordable computational complexity and with performance-guarantee, e.g., the convergence to the global optimum in a finite number of iterations is provable.

In this letter, we investigate this open issue and propose an efficient approach to tackle it. We consider a Poisson cellular network whose resources (spectrum and power) are to be optimally allocated among multiple services (called tenants) so as to maximize the aggregate spectral efficiency under individual performance-guarantee for each tenant. Also, we optimize the scheduling of tenants so as to maximize the aggregate spectral efficiency. By embracing the analytical formulation of the spectral efficiency in [1], we prove that the joint scheduling and resource allocation problem of interest is a Mixed-Integer Non-Linear (MINL) optimization problem, whose global optimum can be calculated by employing the Generalized Benders Decomposition (GBD) [7]. The efficiency of the proposed approach stems from three properties: (i) the spectral efficiency in [1] is a jointly concave function in the transmit power and the bandwidth of the BSs; (ii) the MINL resource allocation problem fulfills the separability conditions in [8]; (iii) the master problem in [7] is linear. Numerical results confirm that the proposed approach offers the same accuracy as brute-force optimization at a similar (or lower) complexity as (than) a sub-optimal greedy algorithm.

\section{Cellular Networks - The Poisson Abstraction}

We consider a single operator downlink cellular network. The locations of the BSs are assumed to constitute a homogeneous PPP of density $\lambda_{\mathrm{BS}}$. Each BS transmits with a constant power $P_{\text {tot }}$ over a spectrum of bandwidth $B_{\text {tot }}$. The 
Telecommunications Operator (TO) offers $T$ different services to its Mobile Terminals (MTs). An MT requests the $i$ th, for $i=1,2, \ldots, T$, service if it wishes to obtain an average data rate at least equal to $\tau_{i}(\mathrm{bit} / \mathrm{s})$. The MTs that request the $i$ th service are collectively referred to as the $i$ th tenant.

The locations of the MTs of the $i$ th tenant are assumed to follow a homogeneous PPP of density $\lambda_{\mathrm{MT}_{i}}$. For example, a tenant may be constituted by the MTs that need to use the network for mobile broadband applications (i.e., $\tau_{i}$ and $\lambda_{\mathrm{MT}_{i}}$ are likely to be large and small, respectively), while another tenant may be constituted by the MTs that need to use the network for sensing applications (i.e., $\tau_{i}$ and $\lambda_{\mathrm{MT}_{i}}$ are likely to be small and large, respectively). In this letter, due to space limitations, we do not consider the case studies of multi-operator cellular networks and services that necessitate other than a minimum guaranteed data rate, e.g., a maximum transmission delay or a minimum number of MTs to be served. These generalizations are postponed to future research.

The locations of the BSs are denoted by $x \in \Psi_{\mathrm{BS}} \subseteq \mathbb{R}^{2}$. The channels of the $T$ tenants and MTs are assumed to be mutually independent and identically distributed. As far as the channels are concerned, therefore, the index $i$ can be omitted. An unbounded path-loss model is considered, i.e., $l(x)=\kappa r^{\alpha}$, where $r=\|x\|$ is the distance of the BS at $x$ from the typical MT, and $\kappa$ and $\alpha>2$ denote the path-loss constant and the path-loss slope, respectively. The power gain of the fastfading is assumed to be an exponential random variable of unit mean. A cell association based on the highest average received power is assumed, which, for the considered path-loss model, is equivalent to the shortest distance cell association.

Let us denote by $P_{i}$ and $B_{i}$ the transmit power and bandwidth that the TO allocates to the $i$ th tenant, respectively. Thus, $P_{i}$ and $B_{i}$ are the power and spectrum that each BS has at its disposal to serve the MTs of the $i$ th tenant associated to it. We assume that the tenants are served over disjoint bands of the spectrum owned by the TO, i.e., $B_{\text {tot }}$, so that no intertenant interference is present. For each tenant, we assume that the BSs equally allocate the bandwidth $B_{i}$ among all the MTs associated to them, and that their transmit power $P_{i}$ is evenly spread across the available bandwidth $B_{i}$. This implies that the considered network model is subject to inter-cell interference, but is not subject to intra-cell interference [1], [5].

Under these modeling assumptions, the resource allocation problem that we are interested in analyzing jointly encompasses: (i) the scheduling of the tenants; and (ii) the optimization of their transmit power, $P_{i}$, and bandwidth, $B_{i}$. In fact, it is not guaranteed that, given $P_{\text {tot }}$ and $B_{\text {tot }}$, the $T$ tenants can achieve the requested minimum rates $\tau_{i}$, for $i=1,2, \ldots, T$, simultaneously. Thus, the optimal sub-set of tenants $(\leq T)$ that maximizes the performance metric of interest (the network's spectral efficiency) needs to be identified. The optimization problem is formulated in the next section, by using recent results on the spectral efficiency and average rate [1].

\section{Problem Statement - An Minl Formulation}

Let $\mathcal{S}_{i}=\mathcal{S}\left(P_{i}, B_{i}, \lambda_{\mathrm{MT}_{\mathrm{i}}} ; \lambda_{\mathrm{BS}}\right)$ and $\mathcal{R}_{i}=$ $\mathcal{R}\left(P_{i}, B_{i}, \lambda_{\mathrm{MT}_{\mathrm{i}}} ; \lambda_{\mathrm{BS}}\right)$ be the network's spectral efficiency and the user's average rate of the $i$ th tenant, respectively. $\mathcal{S}_{i}$ and $\mathcal{R}_{i}$ yield the bit/s/m and the bit/s that the TO is capable of delivering to the $i$ th tenant throughout the network and to the typical MT, respectively. Let $\beta_{i} \in\{0,1\}$ be $0-1$ variables for $i=1,2, \ldots, T$. We assume, by definition, the following relation among $\beta_{i}, P_{i}$, and $B_{i}$ for $i=1,2, \ldots, T$ :

$$
\begin{array}{rr}
\mathcal{S}\left(P_{i}=0, B_{i}=0, \lambda_{\mathrm{MT}_{\mathrm{i}}} ; \lambda_{\mathrm{BS}}\right)=0 & \forall i=1,2, \ldots, T \\
\mathcal{R}\left(P_{i}=0, B_{i}=0, \lambda_{\mathrm{MT}_{\mathrm{i}}} ; \lambda_{\mathrm{BS}}\right)=0 & \forall i=1,2, \ldots, T \\
\beta_{i}=0 \Leftrightarrow P_{i}=0, B_{i}=0 & \forall i=1,2, \ldots, T
\end{array}
$$

The conditions in (1) and (2) imply the physical constraint that $\mathcal{S}_{i}$ and $\mathcal{R}_{i}$ need to be zero if no power and spectrum are allocated to the $i$ th tenant. The condition in (3) allows us to interpret $\beta_{i}$ as an indicator variable that is equal to zero if and only if the $i$ th tenant is not scheduled (or admitted), and, hence, the TO allocates no power and spectrum to its MTs.

The problem (P1) of jointly scheduling the tenants and allocating their resources (power, spectrum) is formulated as:

$$
\text { P1 : } \begin{aligned}
\underset{P_{i}, B_{i}, \beta_{i}}{\operatorname{maximize}} & \sum_{i=1}^{T} \ln \left[\left(1+\beta_{i}\right)\left(1+\mathcal{S}_{i}\right)\right] \\
\text { subject to } & \mathcal{R}_{i} \geq \beta_{i} \tau_{i} \\
& 0 \leq B_{i} \leq \beta_{i} B_{\text {tot }} \\
& 0 \leq P_{i} \leq \beta_{i} P_{\text {tot }} \\
& \sum_{i=1}^{T} B_{i} \leq B_{\text {tot }} \\
& \sum_{i=1}^{T} P_{i} \leq P_{\text {tot }} \\
& \beta_{i} \in\{0,1\}
\end{aligned}
$$

P1 can be interpreted as follows. The objective function in (4) is the aggregate spectral efficiency of all the tenants that can be scheduled. The logarithmic function is employed in order to ensure a certain level of fairness among the tenants [9]. Based on (1)-(3), the objective function is appropriately chosen to be non-negative, i.e., if $\beta_{i}=0$, then $\ln (1)=0$. This is the reason of the presence of the " +1 " terms. If $\beta_{i}=0$, in particular, the $i$ th tenant does not contribute to the objective function. The constraint in (5) ensures that the minimum rate requirement is fulfilled. The constraints in (6) and (7) ensure that the bandwidth and power are non-negative, and the constraints in (8) and (9) that the total allocated spectrum and power do not exceed $B_{\text {tot }}$ and $P_{\text {tot }}$, respectively. In (5)-(7) the presence of the $0-1$ variables $\beta_{i}$ ensures that the constraints are effective only if the $i$ th tenant is scheduled $\left(\beta_{i}=1\right)$. Also, by virtue of (3), and the constraints in (6) and (7), there is no need to add the $0-1$ variables $\beta_{i}$ in (8) and (9).

P1 requires an analytical expression for $\mathcal{S}_{i}$ and $\mathcal{R}_{i}$. For the considered network model, $\mathcal{S}_{i}$ and $\mathcal{R}_{i}$ are defined and formulated in [1, Eq. (7)] and [1, Eq. (9)], and, for completeness, they are reported in the next two lemmas, where: (i) $\gamma_{D}$ is the minimum signal-to-interference ratio in order for the typical MT to decode the data from the serving BS; (ii) $\gamma_{A}$ is the minimum average signal-to-noise ratio in order for the typical MT to detect the pilot signals during the cell association phase; (iii) $N_{0}$ is the receiver's noise spectral density at the typical MT; (vi) $L(x)=1-(1+x / 3.5)^{-3.5}$; and (v) $\Upsilon(y, z)={ }_{2} F_{1}(-2 / z, 1,1-2 / z,-y)-1$, where ${ }_{2} F_{1}(\cdot)$ is the Gaussian hypergeometric function. 
Lemma 1: Consider the network model in Section II. Define $\Phi\left(\lambda_{\mathrm{BS}}, \lambda_{\mathrm{MT}_{i}}, \gamma_{D}, \alpha\right)=1+L\left(\lambda_{\mathrm{MT}_{i}} / \lambda_{\mathrm{BS}}\right) \Upsilon\left(\gamma_{D}, \alpha\right)$. The spectral efficiency of the $i$ th tenant can be formulated as:

$$
\begin{aligned}
& \mathcal{S}_{i}=B_{i} \log _{2}\left(1+\gamma_{D}\right) \frac{\lambda_{\mathrm{BS}} L\left(\lambda_{\mathrm{MT}_{i}} / \lambda_{\mathrm{BS}}\right)}{\Phi\left(\lambda_{\mathrm{BS}}, \lambda_{\mathrm{MT}_{i}}, \gamma_{D}, \alpha\right)} \\
& \times\left(1-\exp \left(-\pi \lambda_{\mathrm{BS}}\left(\frac{P_{i} / B_{i}}{\kappa \gamma_{A} N_{0}}\right)^{2 / \alpha} \Phi\left(\lambda_{\mathrm{BS}}, \lambda_{\mathrm{MT}_{i}}, \gamma_{D}, \alpha\right)\right)\right)
\end{aligned}
$$

Proof: It follows from the definition of $\mathcal{S}_{i}$ in [1, Eq. (7)] and the analytical steps reported in [1, Appendix A].

Lemma 2: Consider the network model in Section II. The user's average rate of the $i$ th tenant is equal to $\mathcal{R}_{i}=\mathcal{S}_{i} / \lambda_{\mathrm{MT}_{i}}$, where $\mathcal{S}_{i}$ is formulated in closed-form in (11).

Proof: It is similar to the proof of Lemma 1 [1].

It is worth mentioning, as detailed in [1, Remark 7], that $\mathcal{S}_{i}$ in Lemma 1 is defined from the viewpoint of the typical MT rather than from the viewpoint of the typical BS. Thus, it is an approximated formulation of the network's spectral efficiency. Besides the proof in [1, Appendix A], this remark justifies the relation between $\mathcal{R}_{i}$ and $\mathcal{S}_{i}$ in Lemma 2 as well.

$\mathbf{P 1}$ is an MINL program and, hence, is NP-hard [8]. Tackling P1 via global optimization methods based on the branch-andbound algorithm is possible, but subject to identifying suitable bounds for its application [8]. To devise an algorithm that is computationally efficient and provably convergent to the global optimum, we need the following two preliminary results.

Proposition 1: The network's spectral efficiency, $\mathcal{S}_{i}$, and the user's average rate, $\mathcal{R}_{i}$, in Lemma 1 and Lemma 2, respectively, are jointly concave functions in $P_{i}$ and $B_{i}$.

Proof: The proof follows by computing the Hessian matrix of $\mathcal{S}_{i}$ (or $\mathcal{R}_{i}$ ) as a function of $P_{i}$ and $B_{i}$, and observing that the corresponding quadratic form is negative semidefinite.

Lemma 3: By conditioning upon the $0-1$ variables $\beta_{i}, \mathbf{P 1}$ is a convex optimization problem.

Proof: The proof follows by noting that, conditioning on the variables $\beta_{i}$, the objective in (4) is a concave function since it is the sum of concave functions by virtue of Proposition 1, the constraint in (5) is a concave function by virtue of Proposition 1 , and the constraints in (6)-(9) are affine functions.

Based on Proposition 1 and Lemma 3, the following conclusions about $\mathbf{P 1}$ can be drawn.

- If no scheduling is considered, $\mathbf{P 1}$ is a convex optimization problem that can be efficiently solved in polynomial time regardless of the number of tenants. This feature originates from the mathematical tractability of the recently proposed definition of coverage probability in [1].

- The complexity of P1 originates from jointly optimizing the scheduling of the tenants, and the power and spectrum of the tenants admitted into the network.

- By virtue of Proposition 1, P1 may be tackled by employing an exhaustive search over the $T$ binary variables $\beta_{i}$, and by applying convex optimization methods to compute, for each possible case, the optimal power and spectrum to be allocated to the corresponding tenants. Even for modest values of the number of services that the TO needs to support, e.g., $T=20$, the computational complexity of such a brute-force method may be pro-

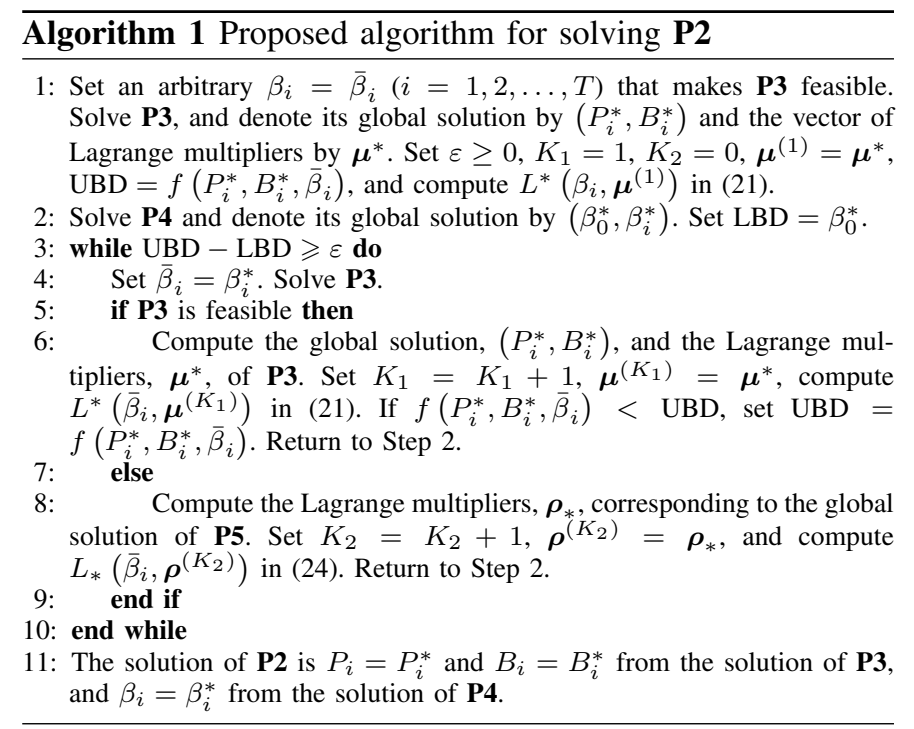

hibitive. Given the need of future networks to overcome the "one-size-fits-all" design paradigm, and to be highly adaptable to support multiple and disparate services, a computationally-efficient algorithm with provable convergence to the global optimum is needed.

In Section IV, we capitalize on Proposition 1 and the structure of the objective and the constraints in (4)-(10) in order to develop an algorithm, based on the GBD, that is provably convergent to the global solution of P1. This cannot be guaranteed, in general, by using general-purpose solvers for MINL problems. The proposed approach has, in general, exponential complexity, but numerical results show that it is faster than a (sub-optimal) greedy algorithm (see Section V).

IV. Algorithmic Solution - GBD-BAsed Approach

The proposed approach for solving P1 is based on the GBD originally introduced in [7]. We show, in particular, that the specific properties of $\mathcal{S}_{i}$ and $\mathcal{R}_{i}$ proved in Proposition 1 allows us to obtain an efficient iterative implementation that necessitates the solution of a few convex optimization problems and Mixed-Integer Linear (MIL) optimization problems, whose global solution can be provably guaranteed.

Define the column-vector of constraints $\mathbf{G}\left(P_{i}, B_{i}, \beta_{i}\right)=$ $\left[G_{1} ; G_{2} ; \ldots ; G_{7}\right]$, whose seven rows are: $G_{1}=\beta_{i} \tau_{i}-\mathcal{R}_{i}$; $G_{2}=B_{i}-\beta_{i} B_{\text {tot }} ; G_{3}=P_{i}-\beta_{i} P_{\text {tot }} ; G_{4}=-B_{i} ; G_{5}=$ $-P_{i} ; G_{6}=\sum_{i=1}^{T} B_{i}-B_{\text {tot }} ;$ and $G_{7}=\sum_{i=1}^{T} P_{i}-P_{\text {tot }}$. Define $f\left(P_{i}, B_{i}, \beta_{i}\right)=f_{1}\left(\beta_{i}\right)+f_{2}\left(P_{i}, B_{i}\right)$, where $f_{1}\left(\beta_{i}\right)=$ $-\sum_{i=1}^{T} \ln \left(1+\beta_{i}\right)$ and $f_{2}\left(P_{i}, B_{i}\right)=-\sum_{i=1}^{T} \ln \left(1+\mathcal{S}_{i}\right) . \mathbf{P 1}$ can be re-written, in canonical form, as follows:

$$
\text { P2: } \begin{aligned}
\underset{P_{i}, B_{i}, \beta_{i}}{\operatorname{minimize}} & f\left(P_{i}, B_{i}, \beta_{i}\right) \\
\text { subject to } & \mathbf{G}\left(P_{i}, B_{i}, \beta_{i}\right) \leq 0 \\
& \beta_{i} \in\{0,1\}
\end{aligned}
$$

Algorithm 1 reports the proposed approach for solving P2. Algorithm 1 is an iterative algorithm that recursively solves the three optimization problems, P3, P4, and P5 defined in the following paragraphs. The optimality and rational of Algorithm 1 are discussed at the end of this section. 
a) Problem P3: P3 is defined as follows:

$$
\begin{aligned}
& \text { P3 : } \underset{P_{i}, B_{i}}{\operatorname{minimize}} f\left(P_{i}, B_{i}, \bar{\beta}_{i}\right) \\
& \text { subject to } \quad \mathbf{G}\left(P_{i}, B_{i}, \bar{\beta}_{i}\right) \leq 0
\end{aligned}
$$

where $\bar{\beta}_{i}$ denotes a given instance of $\beta_{i}(i=1,2, \ldots, T)$, as stated in Algorithm 1. Since the 0-1 variables are not subject to optimization, P3 is a convex problem by virtue of Lemma 3. Thus, its global optimum is obtained in polynomial time.

b) Problem P5: Let $\bar{\beta}_{i}$ be an instance of $\beta_{i}$ that makes $\mathbf{P 3}$ feasible. Let $\theta$ be a real variable. $\mathbf{P 5}$ is defined as:

$$
\begin{array}{ll}
\text { P5 : } \begin{array}{r}
\underset{P_{i}, B_{i}, \theta}{\operatorname{minimize}} \\
\text { subject to }
\end{array} & \mathbf{G}\left(P_{i}, B_{i}, \bar{\beta}_{i}\right)-\theta \mathbf{1} \leq 0
\end{array}
$$

Since the $0-1$ variables $\beta_{i}$ are not to be optimized, $\mathbf{P 5}$ is a convex optimization problem by virtue of Proposition 1 . Let $\boldsymbol{\rho}_{*}=\left[\rho_{i} ; \rho_{0 B_{i}} ; \rho_{0 P_{i}} ; \rho_{00 B_{i}} ; \rho_{00 P_{i}} ; \rho_{B} ; \rho_{P}\right]$ be the columnvector of Lagrange multipliers of $\mathbf{P 5}$, whose eth entry corresponds to $G_{e}(e=1,2, \ldots, 7)$ in (18). $\boldsymbol{\rho}_{*}$ is needed to formulate $\mathbf{P 4}$. It is worth mentioning that $\mathbf{P 5}$ is an equivalent (convex) formulation of [7, Eq. (6)], as elaborated in [10].

c) Problem P4: Before formulating P4, we introduce the auxiliary optimization problems $\mathbf{P} \mathbf{4}^{*}$ and $\mathbf{P} \mathbf{4}_{*}$.

Let $\boldsymbol{\mu}^{*}=\left[\mu_{i} ; \mu_{0 B_{i}} ; \mu_{0 P_{i}} ; \mu_{00 B_{i}} ; \mu_{00 P_{i}} ; \mu_{B} ; \mu_{P}\right]$ be the column-vector of Lagrange multipliers of $\mathbf{P 3}$, whose eth entry corresponds to $G_{e}(e=1,2, \ldots, 7)$ in (16). Let $\bar{b}_{i}=\mu_{0 B_{i}}-$ $\mu_{00 B_{i}}+\mu_{B}$ and $\bar{p}_{i}=\mu_{0 P_{i}}-\mu_{00 P_{i}}+\mu_{P}, \mathbf{P 4}^{*}$ is defined as:

$\mathbf{P 4}^{*}: \underset{P_{i}, B_{i}}{\operatorname{minimize}} \sum_{i=1}^{T}\left(-\ln \left(1+\mathcal{S}_{i}\right)-\mu_{i} \mathcal{S}_{i}+\bar{b}_{i} B_{i}+\bar{p}_{i} P_{i}\right)$

subject to (8) and (9)

Let $f_{\mathrm{P} 4^{*}}$ be the objective function evaluated at the optimal solution of $\mathbf{P 4}^{*}$ in (19). Define the following function ${ }^{1}$ :

$$
\begin{aligned}
& L^{*}\left(\beta_{i}, \boldsymbol{\mu}^{*}\right)=f_{\mathrm{P} 4^{*}}-\mu_{B} B_{\mathrm{tot}}-\mu_{P} P_{\mathrm{tot}} \\
& +\sum_{i=1}^{T} \beta_{i}\left(\mu_{i} \lambda_{\mathrm{MT}_{i}} \tau_{i}-\ln (2)-\mu_{0 B_{i}} B_{\mathrm{tot}}-\mu_{0 P_{i}} P_{\mathrm{tot}}\right)
\end{aligned}
$$

Let $\boldsymbol{\rho}_{*}$ be the column-vector defined in P5. Let $\underline{b}_{i}=\rho_{0 B_{i}}-$ $\rho_{00 B_{i}}+\rho_{B}, \underline{p}_{i}=\rho_{0 P_{i}}-\rho_{00 P_{i}}+\rho_{P} . \mathbf{P} \mathbf{4}_{*}$ is defined as follows:

$$
\begin{aligned}
\mathbf{P 4}_{*} & \underset{P_{i}, B_{i}}{\operatorname{minimize}} \sum_{i=1}^{T}\left(-\rho_{i} \mathcal{S}_{i}+\underline{b}_{i} B_{i}+\underline{p}_{i} P_{i}\right) \\
& \text { subject to (8) and (9) }
\end{aligned}
$$

Let $f_{\mathrm{P} 4_{*}}$ be the objective function evaluated at the optimal solution of $\mathbf{P} \mathbf{4}_{*}$ in (22). Define the following function:

$$
\begin{aligned}
L_{*}\left(\beta_{i}, \boldsymbol{\rho}_{*}\right) & =f_{\mathrm{P} 4_{*}}-\rho_{B} B_{\mathrm{tot}}-\rho_{P} P_{\mathrm{tot}} \\
& +\sum_{i=1}^{T} \beta_{i}\left(\rho_{i} \lambda_{\mathrm{MT}_{i}} \tau_{i}-\rho_{0 B_{i}} B_{\mathrm{tot}}-\rho_{0 P_{i}} P_{\mathrm{tot}}\right)
\end{aligned}
$$

By virtue of Proposition $1, \mathbf{P 4}^{*}$ and $\mathbf{P} \mathbf{4}_{*}$ are convex and their global solution can be computed in polynomial time.

Let $\beta_{0}$ be a real number. $\mathbf{P 4}$ is defined as follows:

$$
\text { P4 : } \underset{\beta_{0}, \beta_{i}}{\operatorname{minimize}} \quad \beta_{0}
$$

${ }^{1}$ The $\ln (2)$ term is obtained by noting that $\ln \left(1+\beta_{i}\right)=\beta_{i} \ln (2)$.

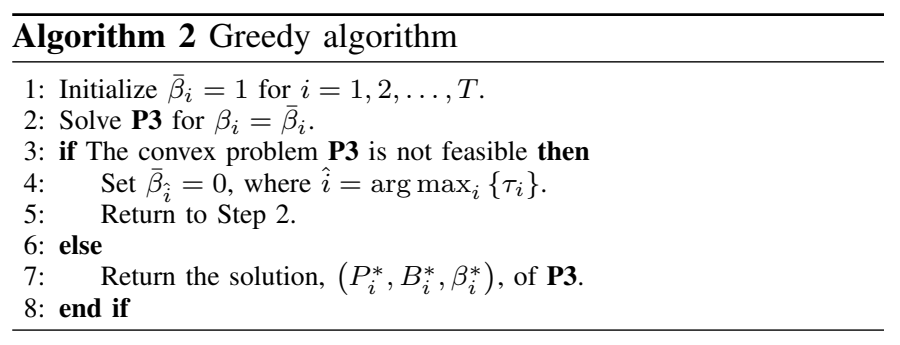

$$
\begin{array}{ll}
\text { subject to } & L^{*}\left(\beta_{i}, \boldsymbol{\mu}^{\left(k_{1}\right)}\right) \leq \beta_{0} \quad k_{1}=1,2, \ldots, K_{1} \\
& L_{*}\left(\beta_{i}, \boldsymbol{\rho}^{\left(k_{2}\right)}\right) \leq 0 \quad k_{2}=1,2, \ldots, K_{2}
\end{array}
$$

where $\boldsymbol{\mu}^{\left(k_{1}\right)}=\boldsymbol{\mu}^{*}$ and $\boldsymbol{\rho}^{\left(k_{2}\right)}=\boldsymbol{\rho}_{*}$ as defined in Algorithm 1.

$\mathbf{P 4}$ is referred to as the master problem. The master problem in (25)-(27) is, in particular, an MIL optimization problem, whose global solution can be computed by using the branchand-cut algorithm based on Gomory cuts [11]. More specifically, a sequence of continuous relaxations of $\mathbf{P 4}$ are solved by using standard linear programming methods $[8$, Chapter 5]. New Gomory cuts are added each time that the solution of $\mathbf{P 4}$ results in some variables that are not integer numbers. It is proved in [11, Theorem 9.13] that the branch-and-cut algorithm based on Gomory cuts applied to an MIL converges to its global solution in a finite number of iterations.

Therefore, three remarks about Algorithm 1 can be made.

- In contrast to P2, the computation of the global solution of $\mathbf{P 4}$ can be guaranteed in a finite number of iterations. The reason is that $\mathbf{P 4}$ is a linear optimization problem and a systematic procedure to compute the cuts, which assures the convergence to the global optimum, exists.

- Algorithm 1 consists of iteratively solving convex and MIL optimization problems. Since all the considered optimization problems can be solved globally, Algorithm 1 provably converges to the optimum of $\mathbf{P 2}$ in a finite number of iterations [7, Theorem 2.5]. In particular, Algorithm 1 is $\varepsilon$-optimal (with $\varepsilon>0$ ).

- Since P4 is a non-convex optimization problem, it is not possible to ensure that the global optimum of Algorithm 1 is computed in polynomial time. In Section V, we show, however, that Algorithm 1 is more efficient than other competing algorithms, including solving P1 by using branch-and-bound methods applied to MINL problems.

\section{NumericAl RESUlTS}

In this section, we substantiate the global optimality and analyze the complexity of Algorithm 1. With the aid of the OPTI toolbox integrated in Matlab [12], P3, P5, P4*, and $\mathbf{P 4}_{*}$ are solved by using the IPOPT solver, which employs the primal-dual interior point method, and $\mathbf{P 4}$ is solved by using the SCIP solver, which employs the branch-and-cut method.

We compare Algorithm 1 against three benchmark algorithms: (i) the exhaustive search (brute-force) approach described in Section III, which consists of solving $2^{T}$ convex optimization problems and then computing the best solution among them. The convex problems are solved by using the IPOPT solver of the OPTI toolbox; (ii) the branch-and-bound algorithm, which is directly applied to the MINL optimization 
TABLE I: Simulation setup

\begin{tabular}{l}
\hline \hline$\lambda_{\mathrm{BS}}=1 /\left(\pi \mathrm{R}_{\text {cell }}^{2}\right), \lambda_{\mathrm{MT}}=1 /\left(\pi \mathrm{R}_{\mathrm{MT}}^{2}\right), R_{\text {cell }}=500 \mathrm{~m}$, \\
$\gamma_{I}=\gamma_{A}=10 \mathrm{~dB}, \kappa=38.9 \mathrm{~dB}, \alpha=3.5, T=20$, \\
$B_{\mathrm{tot}}=100 \mathrm{MHz}, P_{\mathrm{tot}}=30 \mathrm{dBm}, N_{0}=-174 \mathrm{dBm} / \mathrm{Hz}$, \\
$\tau_{1: 7}=37.74,885.17,913.29,796.18,98.71,261.87,335.36$ \\
$\tau_{8: 14}=679.73,136.55,721.23,106.76,653.76,494.17,779.05$ \\
$\tau_{15: 20}=715.04,903.72,890.92,334.16,698.75,197.81$ \\
$\mathrm{R}_{\mathrm{MT}_{1: 7}}=5.13,15.75,48.06,1.75,55.73,43.82,29.32$ \\
$\mathrm{R}_{\mathrm{MT}_{8: 14}}=34.71,14.24,27.53,57.79,32.81,31.27,13.90$ \\
$\mathrm{R}_{\mathrm{MT}_{15: 20}}=29.33,37.44,40.75,23.73,22.05,59.28$ \\
\hline \hline
\end{tabular}

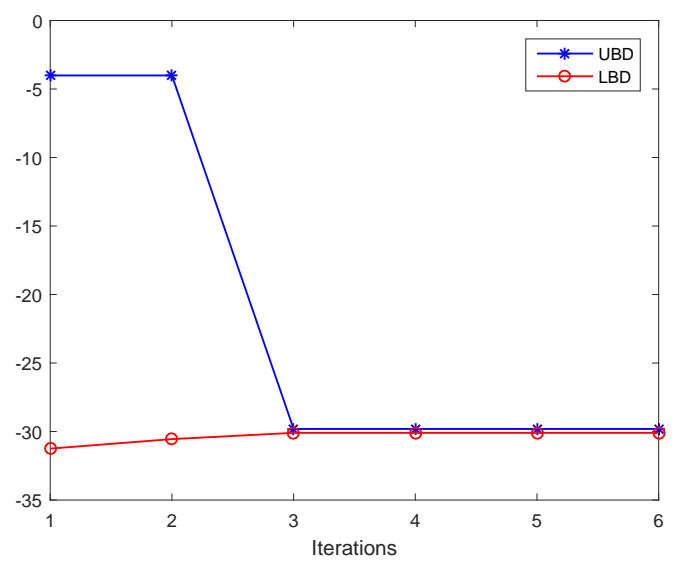

Fig. 1: Convergence of Algorithm 1.

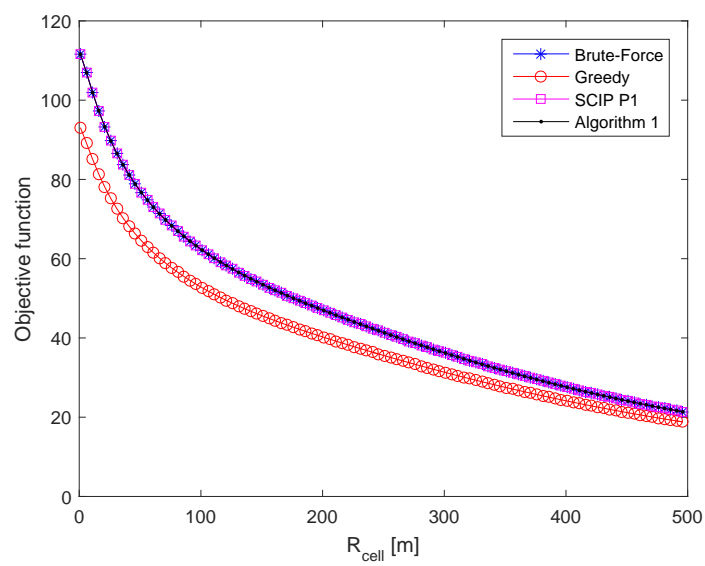

Fig. 2: Comparison with benchmarks: Objective function.

problem P1 (equivalently P2). In this case, for a fair comparison with Algorithm 1, the SCIP solver of the OPTI toolbox is applied; and (iii) the greedy-like Algorithm 2.

The simulation setup is given in Table I. The numerical results are obtained on a Lenovo Thinkpad T440 platform (Intel Core i7-4500U, CPU 1.80-2.40 GHz, RAM 8.00 GB).

In Fig. 1, the convergence of Algorithm 1 is analyzed. In particular, the UBD and LBD functions defined in Algorithm 1 are plotted as a function of the iterations. The gap between the UBD and LBD functions decreases with the number of iterations, which confirms the convergence of Algorithm 1.

In Fig. 2, the objective function in (4) that corresponds to the solution of Algorithm 1 is compared against the other three benchmark algorithms. Algorithm 1 returns the same objective function as the brute-force search and the SCIP general-purpose solver applied to $\mathbf{P 2}$, while Algorithm 2 yields worse performance due to its sub-optimality. Therefore, it is confirmed that algorithm 1 returns the global optimum.

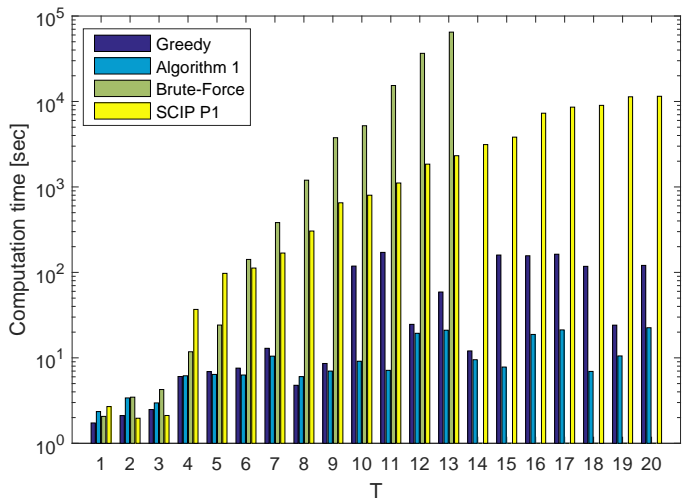

Fig. 3: Comparison with benchmarks: Computation time.

The advantage of Algorithm 1 compared with brute-force and SCIP algorithms is apparent in Fig. 3. Since Algorithm 1 iteratively solves convex and MIL optimization problems, its computation time is lower than the considered benchmark algorithms. In the considered setup, Algorithm 1 may be even faster, for large value of $T$, than the greedy algorithm. This is due to the small number of iterations that are needed for convergence (around 3, as shown in Fig. 1). The computation time of Algorithm 1 does not increase exponentially with $T$.

\section{CONCLUSION}

We have formulated a mixed-integer non-linear optimization problem based on utility functions obtained from stochastic geometry. By capitalizing on the generalized Benders decomposition, we have devised an efficient numerical algorithm that is provably convergent to the optimum in a finite number of iterations. Numerical simulations confirm the convergence to the same solution as a brute-force search but with reduced computational complexity. The proposed approach constitutes a viable and promising method for solving scheduling and resource allocation problems in Poisson cellular networks.

\section{REFERENCES}

[1] M. Di Renzo et al., "System-level modeling and optimization of the energy efficiency in cellular networks - A stochastic geometry framework", IEEE Trans. Wireless Commun., vol. 17, no. 4, Apr. 2018.

[2] J. G. Andrews et al. "A tractable approach to coverage and rate in cellular networks", IEEE Trans. Commun., vol. 59, no. 11, Nov. 2011.

[3] M. Di Renzo et al., "Inhomogeneous double thinning - Modeling and analysis of cellular networks by using inhomogeneous Poisson point processes", IEEE Trans. Wireless Commun., vol. 17, no. 8, Aug. 2018.

[4] M. Di Renzo et al., "The intensity matching approach: A tractable stochastic geometry approximation to system-level analysis of cellular networks", IEEE Trans. Wireless Commun., vol. 15, no. 9, Sep. 2016.

[5] M. Di Renzo et al., "A tractable closed-form expression of the coverage probability in Poisson cellular networks", IEEE Wireless Commun. Lett., vol. 1, no. 1, pp. 249-252, Feb. 2019.

[6] M. Di Renzo et al., "Spectral-energy efficiency Pareto front in cellular networks: A stochastic geometry framework", IEEE Wireless Commun. Lett., vol. 8, no. 2, pp. 424-427, Apr. 2019.

[7] A. Geoffrion, "Generalized Benders decomposition", Journal of Optimization Theory and Applications, vol. 10, no. 4, 1972.

[8] C. A. Floudas, Nonlinear and Mixed-Integer Optimization: Fundamentals and Applications, Oxford University Press, 1995.

[9] F. Kelly, "Charging and rate control for elastic traffic", European Trans. Telecommun., vol. 8, no. 1 pp. 33-37, Jan.-Feb. 1997.

[10] J. K. Peng et al., "On the tuning of predictive controllers: Application of generalized Benders decomposition to the ELOC Problem", Computers \& Chemical Engineering, vol. 82, pp. 105-114, Jul. 2015.

[11] S. O. Krumke, Integer Programming Polyhedra and Algorithms, 2006.

[12] OPTI Toolbox. Available: https://inverseproblem.co.nz/OPTI/. 